\title{
MedienPädagogik
}

Zeitschrift für Theorie und Praxis der Medienbildung

Themenheft Nr. 37: Medienpädagogik als Schlüsseldisziplin in einer mediatisierten Welt. Perspektiven aus Theorie, Empirie und Praxis Herausgegeben von Henrike Friedrichs-Liesenkötter, Lara Gerhardts, Anna-Maria Kamin und Sonja Kröger

\section{Medienpädagogik und Hochschuldidaktik}

\section{Einblicke zu professionsbezogenen und persönlichen Verwebungen}

Diana Bücker und Iris Neiske

\section{Zusammenfassung}

Der vorliegende Beitrag entstand aus der Motivation heraus, Verwebungen der beiden Berufs- und Handlungsfelder Medienpädagogik und Hochschuldidaktik in ihrer professionstheoretischen Entwicklung und ihrer konkreten Verbindung im Kontext des digitalen Lehrens und Lernens an Hochschulen allgemein und im Besonderen an der Universität Paderborn darzustellen. Der Beitrag setzt einzelne theoretische und praktische Schlaglichter und fokussiert dabei die besondere Bedeutung von Kooperationen zwischen den Bereichen.

\section{Academic Development and Media Education}

\begin{abstract}
The article was based on the motivation to picture the interweaving of media education and Academic Development in the context of digital teaching and learning at universities in particular at the University of Paderborn. The contribution was motivated by the idea to present the interweaving of the two
\end{abstract}


professional and action fields of media pedagogy and didactics in higher education in their profession-theoretical development and their concrete connection in the context of digital teaching and learning at universities, in particular at the University of Paderborn. The contribution highlights individual theoretical and practical aspects and focuses on the special importance of cooperation between the areas.

\section{Einstieg und Motivation}

Immer mehr erfüllen Medien grundlegende Funktionen im Prozess von Erziehung und Sozialisation sowie von Lehren und Lernen. Die Medienpädagogik als wissenschaftliche Disziplin und als Berufsfeld setzt sich in diesen über die gesamte Lebensspanne hinweg bedeutsamen Prozessen, reflexiv mit Fragen der pädagogischen Bedeutung auseinander (vgl. u.a. Tulodziecki 2011.; Hartung-Griemberg und Schorb 2017). Die Integration insbesondere digitaler Medien als (Lernarchitekturen〉 für Prozesse des Forschen und Lernens an Hochschulen (vgl. Schiefner-Rohs und Kerres 2012) stellen ein zentrales Handlungsfeld der Medienpädagogik dar. An diesem Punkt ergeben sich Schnittmengen mit der Hochschuldidaktik, welche als anwendungsorientierte Disziplin auf die theoretische Erforschung und praktische Verbesserung der Lehr- und Lernprozesse im gesamten Hochschulbereich abzielt und ein geeignetes Wissens- und Handlungsrepertoire bereitstellt (Helmke und Schrader 2010, 273; Eckstein 1972, 12).

Der vorliegende Beitrag stellt Verwebungen dieser beiden Berufs- und Handlungsfelder anhand der Diskurse um Professionalisierung und Digitalisierung in der Hochschullehre dar und ergänzt theoretische Perspektiven um ausgewählte Beispiele aus der Praxis an der Universität Paderborn. Einen entscheidenden Beitrag zur Verknüpfung und wechselseitigen Befruchtung der beiden Disziplinen leistete Dorothee Meister, insofern greift der Beitrag auf Projekte und Konzeptionierungsbeispiele ihrer Vita zurück. Dabei erhebt der Beitrag keineswegs Anspruch auf eine allumfassende theoretische Analyse und Gegenüberstellung der beiden multidisziplinären Bereiche. Vielmehr sollen einzelne Schlaglichter gesetzt werden, die ganz im Sinne eines Beitrags für eine Festschrift neue Perspektiven eröffnen sollen. 
In einem ersten Schritt werden die historische Entwicklung der beiden Bereiche kurz rekonstruiert und bestehende professionstheoretische Überlegungen zusammengeführt. Als gemeinsame Herausforderung betrachtet der Beitrag wie medienpädagogische und hochschuldidaktische Veränderungsprozesse nachhaltig begleitet werden können und fokussiert dabei die besondere Bedeutung von Akteurskonstellationen und Kooperationen.

\section{Entwicklung und Professionalisierung von Medienpädagogik und Hochschuldidaktik - eine grobe Skizze zur Einordnung}

Die Wurzeln von Medienpädagogik und Hochschuldidaktik lassen sich weit zurückdatieren. ${ }^{1}$ Mit jedem Aufkommen bis Dato noch unbekannter Medien oder neuer (unkonventioneller) Positionen und Theorien im Bereich der Bildung gab es Bemühungen, sich mit den damit einhergehenden Veränderungen und dadurch ergebenden pädagogischen und didaktischen Konsequenzen auseinanderzusetzen (Meister 2005, 3). Die Reaktionen fielen dabei vielfältig aus. Von euphorisch über pragmatisch bis hin zu verweigernd. Es gab Akteure, die Veränderungsprozesse forcierten und Akteure, die Widerstände und Bedenken zeigten (Meister 2018, 1; 2005, 8).

Die heutige Medienpädagogik und Hochschuldidaktik als Berufsfelder und Disziplinen können als Ergebnis von (pädagogischen) Reaktionen auf verschiedenste gesamtgesellschaftliche Entwicklungen gesehen werden. So entwickelte sich der Begriff der Medienpädagogik aus dem Bestreben heraus,

«einen allgemeinen Begriff für die pädagogische Auseinandersetzung mit dem immer größer gewordenen Spektrum an Massenmedien zu finden» (Tulodziecki 2011, 12).

1 Dieser Beitrag dient nicht einer intensiven historischen Diskussion, daher sei für die Medienpädagogik auf Schorb (1995) und Süss, Lampert, und Wijnen (2013) und für die Hochschuldidaktik auf Dany (2007) und Huber (1983), Bücker (i.V.) verwiesen. Vielmehr soll auf Gemeinsamkeiten in der Entwicklung hingewiesen werden, die bei der zumeist getrennten Betrachtung der beiden Handlungsfelder wenig offensichtlich erscheinen. 
Die Hochschuldidaktik sowie Ideen zur Hochschuldidaktik werden als «Antwort- und Korrekturversuche gegenüber jeweils problematisch gewordenen realen Zuständen und Entwicklungen in der Hochschule» analysiert (Huber 1983, 127). Die Wahl des Begriffs der Hochschuldidaktik fiel in den Reformdiskussionen der 6oer Jahre in der BRD, ausgelöst durch die Ablehnung einer pädagogischen Einflussnahme der Universität auf die Studierenden (ebd., 123).

Bis dahin zumeist noch unter Stichworten wie bspw. Filmkunde, Hodegetik, Hodegese, Methodik des akademischen Studiums, datieren historische Rückblicke die Verwendung von Medienpädagogik und Hochschuldidaktik als Begriffe die jüngere Vergangenheit der 1960er bis 70er Jahre. Trotz der Einführung und verbreiteten Nutzung der Signen sind begriffliche Unklarheiten und Überschneidungen nachweisbar. Bestimmungsversuche werden bis heute von kritischen Diskussionen begleitet, welche die Teilbegriffe (Medien-)Pädagogik und (Hochschul-)Didaktik und die jeweiligen Auslegungen betreffen. Für die Medienpädagogik betrifft dies insbesondere den Diskurs, ob es um die Nutzung der Medien oder die Thematisierung der Medien als Gegenstand geht. Überschneidungen gibt es mit Begriffen wie Medienkunde, Mediendidaktik, Medienbildung und -erziehung, die mal komplementär, mal synonym, mal als Ordnungssystem verstanden werden wobei sich. letzteres durchgesetzt hat (Tulodziecki 2011, 12-13). Für die Hochschuldidaktik betrifft diese Kritik den Begriff der Didaktik, der missverständlich sowie Aus- und Abgrenzungen suggerierend erscheint (u.a. Huber 1983, 116). Damit einher geht das Ringen um Akzeptanz eines möglichst breiten Verständnisses der beiden Handlungsfelder, das über mikrodidaktische Überlegungen hinaus geht und auf eine Integration medienpädagogischer und hochschuldidaktischer Konzepte auf allen Ebenen der jeweiligen Gegenstandsbereiche abzielt. Aktuelle Diskurse beziehen daher dezidiert die Begriffe der Hochschul- und Medienbildung sowie Hochschul- und Medienentwicklung ein².

Innerhalb der Entwicklungen von Medienpädagogik und Hochschuldidaktik lassen sich verschiedene Phasen bzw. Arbeitsansätze nachweisen.

2 Diese Diskurse wurden bei der Konzeptionierung und Gründung der Stabsstelle Hochschuldidaktik und Bildungsinnovationen 2009 an der Universität Paderborn zu Grunde gelegt. Durch verschiedene Drittmittelprojekte wurde diese Stabsstelle kontinuierlich weiterentwickelt. 
Die Medienpädagogik wird in chronologischer Reihenfolge in normative und bewahrpädagogische Ansätze (Beginn 20. Jhd.), instrumentalisierende und ideologisierende Zwecke (1930er), präventive und aufklärende Ausrichtungen (50er), kritisch emanzipatorische, technologisch funktionale (6oer) sowie reflexive und handlungsorientierte Medienkonzepte (70er) differenziert. Während sich hier die Ansätze in zeitlicher Abfolge leichter anordnen lassen, aber auch in heutigen Diskussionen komplementär anzutreffen sind, werden für die Hochschuldidaktik grundlegende Ausrichtungen als parallele sich konkurrierende Konzeptualisierungen benannt (Süss, Lampert, und Wijnen 2013, 95ff.; Huber 1983; Bücker i.V.). Huber differenziert in folgende sechs Arbeitsansätze: unterrichtstechnologisch, sozialpsychologisch, curricular, wissenschaftstheoretisch, berufspraxisbezogen und sozialisationstheoretisch. Er führt weiter aus, dass mit diesen Strömungen dahinterstehende (politische) Ziele und Selbstverständnisse der einzelnen Akteure in Beziehung zu setzen sind: Huber benennt die drei als technologische, funktionale oder kritisch-emanzipatorische Rationalisierung, Eckstein (2012) differenziert in pragmatischem und theoretischem Vorgehen.

Die enge Verzahnung mit gesellschaftlichen Entwicklungen, besonders politischen und ökonomischen Konjunkturen ist beiden Handlungsfeldern inhärent (vgl. u.a. Schorb 1995, 15; Wildt 2013; Bücker i.V.). Daraus ergibt sich eine historische Entwicklung beider Bereiche, die gekennzeichnet ist durch eine wechselhafte Konjunktur, immer abhängig vom Stellenwert und der (gesellschaftlichen) Verantwortung, der ihnen zugeschrieben wird oder von den jeweiligen Vertreterinnen und Vertretern eingefordert wird (vgl. u.a. Schorb 1995, 15; Wildt 2013, 27; Bücker i.V.). Phasen von Ablehnung und Annäherung wechselten sich ab (Hüther und Podehl 2005, 116 zitiert nach Süss, Lampert, und Wijnen 2013, 62).

Dadurch wurde

«eine Vielfalt von Zugängen und Fragestellungen, von Ideen und Konzepten, Theorien und Methoden sowie von Konnotationen und Kontexten in unterschiedlichen Organisationsformen und Institutionalisierungen, Projekten, informellen Handlungszusammenhängen und individuellem Engagement hervorgebracht» (Wildt 2013, 27). 
Sowohl der Medienpädagogik als auch der Hochschuldidaktik wird trotz ihrer bisherigen (Weiter-)Entwicklungen und Professionalisierungstendenzen attestiert, 〈sich in einer Selbstfindungsphase〉 zu befinden und noch offene Fragen nach dem eigenen Selbstverständnis zu haben (Meister und Urban 2014; Stolz und Bücker 2018; Süss, Lampert, und Wijnen 2013, 15).

Diese Diversifikation und Uneindeutigkeit führt zu der Frage, wie die Entwicklung der Handlungsfelder vor dem Hintergrund professionstheoretischer Überlegungen zu deuten sind. Als erziehungswissenschaftliches Kernthema fördert der Diskurs um Profession, Professionalisierung und Pädagogische Professionalität auch in den Bereichen der Medienpädagogik und der Hochschuldidaktik theoretische Überlegungen und empirische Erkenntnisse zu Tage (Scholkmann u.a. 2018; Hugger 2008; diverse Jahrbücher Medienpädagogik u.w.) $)^{3}$ Dabei stehen besonders Fragen nach der Bezugsdisziplin, der Institutionalisierung, der beruflichen Aus- und Weiterbildung, die wissenschaftliche Fundierung des Handelns und das professionelle Selbstverständnis der Akteure im Vordergrund der Überlegungen. Indes ist sich die Community noch uneins, was professionelles Handeln konkret für die jeweiligen Handlungskontexte bedeutet (Stolz und Bücker 2018). Einen besonderen Stellenwert kommen hier dem Theorie-Praxis-Verhältnis und den beruflichen Handlungsdilemmata zu. Eine notwendige professionstheoretische Reflexion der strukturellen Kennzeichen des Berufs auf der einen Seite steht einer gleichzeitigen tatsächlichen Verberuflichung auf der anderen Seite gegenüber. Gleichzeitig muss konstatiert werden, dass es beiden Feldern an einer notwendigen Nachhaltigkeit der bisherigen Massnahmen fehlt. Weiter geht auch der gesellschaftliche Diskurs über Willenserklärungen nicht grundlegend hinaus (Süss, Lampert, und Wijnen 2013; Hugger 2008, 555 u. 565; Stolz und Bücker 2018; Meister und Urban 2014; Meister 2018, 2).

So schliesst sich die Frage an, wie Innovationsprozesse durch medienpädagogische und hochschuldidaktische Massnahmen angestossen und nachhaltige Veränderungsprozesse erreicht werden können. Der folgende Abschnitt fokussiert dabei spezifische Akteurskonstellationen und das strategische Handeln der Akteure im Berufsfeld als zwei exemplarische Faktoren.

3 Für den Diskurs um das Verhältnis zur Erziehungswissenschaft sei u.a. auf Huber und Reiber (2017) verwiesen. 


\section{Veränderungsprozesse an Hochschulen anstossen - Akteurskonstellationen, Kooperation und strategisches Handeln}

Als Pioniere bzw. Erstnutzer greifen Hochschulen auf Medien als Mittel zur Kommunikation von Wissen zurück. Als essenzieller Bestandteil der Arbeitsumgebung von Wissenschaftlerinnen und Wissenschaftlern und Studierenden in Forschung und Verwaltung, erfolgt der Einzug der $\mathrm{Me}-$ dien im Bereich der Lehre indes eher zögerlich und teilweise mit grossem Vorbehalt (vgl. (Schiefner-Rohs und Kerres 2012, 2). Dies verwundert, da (digitale) Medien als zentrales didaktisches Handlungsfeld innerhalb der Kernaufgabe Lehren und Lernen verstanden werden können (Kamin und Meister 2015, 8).

Die nachhaltige Integration medienpädagogischer und hochschuldidaktischer Konzepte in die Hochschule erfordert ein komplexes Zusammenspiel verschiedener Akteurinnen und Akteuren aus Studium und Lehre. Dies gilt sowohl auf der Mikro- als auch auf der Makroebene, auf individueller wie gesamtuniversitärer Ebene.

Den einzelnen Lehrenden wird dabei häufig eine Gatekeeper Funktion zugeschrieben, von denen die langfristige Umsetzung neuer Projekte abhängt. Für den Einsatz digitaler Medien zitieren Schiefner-Rohs und Kerres (2012):

«Den Lehrenden, ihrer Kompetenz und Motivation kommt eine Schlüsselrolle zu, um E-Learning zu verankern und eine innovative Lehr-LernKultur zu etablieren. Die Gewinnung einer größeren Menge von Lehrenden für E-Learning-Innovationen macht ein Faculty Engagement (Hagner/Schneebeck 2001) notwendig und Maßnahmen, die an den Bedingungen dieser Zielgruppe ansetzen» (Schiefner-Rohs und Kerres 2012, 12 mit Verweis auf Seufert und Euler 2005).

Ein kommunikativ-kooperativer Prozess zwischen den Beteiligten gilt auch für die Hochschuldidaktik als notwendig:

«Die Entwicklung von Lehre vor dem Hintergrund hochschuldidaktischen Wissens ist keine Ware, die von Hochschuldidaktiker*innen produziert und an die Hochschullehrenden und für Studium und 
Lehre Verantwortlichen weitergegeben wird, sondern eine Leistung, die in einem kommunikativ-kooperativen Prozess zwischen Hochschuldidaktiker*innen und Hochschullehrenden entsteht» (Stolz und Bücker 2018; Stolz, im Druck).

Doch die Zusammenarbeit von Hochschul- und Mediendidaktikern mit weiteren Akteuren scheint herausfordernd. Angestrebte Veränderungsprozesse treffen auf eine auffallend starke Beharrung auf traditionelle und routinierte Lehr- und Lernverfahren und ein direktes Einwirken auf Lehrpersonen scheint nicht möglich. Stolz (i.V.), Schiefner-Rohs und Kerres (2012) und Bücker (i.V.) sehen die Gründe unter Anderem in dem Spannungsfeld von spezifischen Auffassungen hinsichtlich der Notwendigkeit von Veränderungen, den sich daraus ergebenden strategischen Handlungsansätzen auf der Einen und der hohen Autonomie der Lehrpersonen auf der anderen Seite.

Didaktisches Handeln kann - je nach Akteurs- und Projektkonstellation - als Eingriff oder gar Nötigung aufgefasst werden (Schiefner-Rohs und Kerres 2012). «Wird 〈das Neue〉 zu sehr als Veränderung als Reform oder gar Revolution angekündigt, kann dies die Menschen abschrecken» (Schiefner-Rohs und Kerres 2012, 6, Herv. i. Original). Als notwendige Strategien werden die «allparteiliche Kooperation» (Szczyrba, Heuchemer, und van Treeck 2012), die «Kooperationsherstellung» (Stolz in Druck) sowie ein Active Change, welcher mit vielen Gesprächen, Informationen und Beratungen verbunden ist (Schiefner-Rohs und Kerres 2012,6) genannt. Weiter fällt der Begriff einer Kultur des Ermöglichens, «[...] denn Ermöglichen ist nicht Herstellen, sondern etwas, das in Interaktion entsteht - jemand oder etwas ermöglicht etwas mit oder für jemand anderen» (Gotzen, Heuchemer, und Treeck 2019). Solche Fragen nach den Momenti und den zentralen Akteurskonstellationen, die eine erfolgreiche Verbreitung und nachhaltige Verankerung als Qualitätssicherungsmassnahme von Digitalisierungsprojekten innerhalb der Digitalisierungsstrategie ausmachen, stehen im i Projekt QuaSiD im Fokus (Graf-Schlattmann u.a. 2018).

Exemplarisch für ein Format, welches auf eben diese Kooperation und Interaktion abzielt, kann die unter Dorothee Meisters Federführung initiierte〈LernPause〉 genannt werden. 


\section{Die 〈LernPause)}

Mit dem Ziel das Digitale Lehren und Lernen als festes Gesprächsthema an der Universität Paderborn zu verankern fand im Februar 2010 ein erstes Paderborner Werkstattgespräch zum Thema 〈E-Learning und Lehre〉 statt - später in 〈LernPause〉 umbenannt. Das Besondere an dieser Veranstaltung ist, dass sie Institutsübergreifend angelegt ist, und von der Vizepräsidentin oder dem Vizepräsidenten für Lehre, Studium und Qualitätsmanagement, der Stabsstelle Bildungsinnovationen und Hochschuldidaktik sowie dem Zentrum für Informations- und Medientechnologien (IMT) organisiert wird.

Das Angebot verbindet das Mittagessen mit einem niedrigschwelligen Fortbildungsangebot. Durch fachliche Impulse zum Thema E-Learning finden sich immer neue Konstellationen von Lehrenden, die die Möglichkeit zum konstruktiven und doch auch informellen Austausch zum Thema Lehre nutzen. Early Adopters treffen auf Early Majority - die Gruppe, deren Innovationsentscheidungsprozess länger dauert, die aber besser zu überzeugen sind und schneller Innovationen umsetzten als die Late Majority. Studierende wie Professoren werden gleichermassen gehört und beteiligt (Budde, Oevel, und Neiske 2017, 1750).

Wie können Hochschulen nun also die nachhaltige Integration von ELearning und hochschuldidaktischen Ansätzen forcieren? Hochschulen brauchen

«übergeordnete Strategien für das digitale Zeitalter, um zeitgemäße Lehr- und Lernformate, Beratungs- und Unterstützungsangebote sowie neue Curriculums- und Studienstrukturen an den Zielen ihrer Hochschulentwicklung, den Zielgruppen der Hochschule und ihrem Profil auszurichten» (Hochschulforum Digitalisierung 2017).

Das Hochschulforum Digitalisierung, dem auch Dorothee Meister seit 2014 angehört, hebt dabei die Notwendigkeit einer individuell angepassten Strategie hervor:

«Einen einheitlichen Weg dem digitalen Wandel zu begegnen kann es aufgrund der Diversität und Heterogenität der deutschen Hochschulen und der Vielfalt digitaler Lehr- und Lernszenarien dabei kaum geben.» (Hochschulforum Digitalisierung 2017). 
Will man klären, was alles unter einer solchen Strategie zu fassen ist, so lässt sich für diesen Beitrag pointiert zusammenführen: Strategieentwicklung ist als Top-Down und Bottom-Up Ansatz zu beschreiben (GrafSchlattmann u.a. 2018; Schiefner-Rohs und Kerres 2012). Darüber hinaus kann festgehalten werden «was eine Hochschulstrategie - aller Wahrscheinlichkeit nach - nicht ist: Ein Plan, der von der Hochschulleitung entworfen und stringent innerhalb der Arbeitseinheiten durchgeführt wird» (Graf-Schlattmann u.a. 2018, 34).

\section{Projekte der Hochschulentwicklung an der Universität Paderborn}

Eine Hochschulstrategie vielmehr als gesamtuniversitären Hochschulentwicklungsprozess zu definieren, führte unter Mitwirkung von Dorothee Meister auch an der Universität Paderborn zu erfolgreichen Projekten. Benannt seien an dieser Stelle

1. das Projekt «Locomotion» (Low-Cost Multimedia Organisation und Production), welches das Ziel verfolgte, eine prozessbasierte Unterstützungsumgebung für E-Learning aufzubauen (Hauenschild, Meister, und Schäfer 2010),

2. das Projekt «Heterogenität als Chance», welches im Rahmen der Qualitätspakt Lehre Förderlinie des BMBF, den gesamten Student-Life-Cycle und die universitäre Lehre in den Blick nimmt (Riegraf u.a. 2018) sowie

3. das Projekt Innoversity, welches im Rahmen des Wettbewerbs «Hochschulbildung und Digitalisierung» des Stifterverbands darauf abzielte, durch ein Innovationsmanagement einzelne Projektideen nachhaltig zu verankern (Budde, Oevel, und Neiske 2017).

Den Projekten ist inhärent, dass in verschiedenen Massnahmenbündeln Hochschulbildung und Digitalisierung zusammen gedacht wurden. Die Schnittstellen von Hochschuldidaktik und E-Learning wurden genutzt und allparteiliche Kooperation durch strukturelle wie inhaltliche Förderung forciert. Die jeweiligen Antragsphasen waren als Bottom-up Prozess angelegt und wurden gemeinsam mit allen Fakultäten und Statusgruppen realisiert. 


\section{Fazit}

Der Beitrag zeigte erste Berührungspunkte zwischen Medienpädagogik und Hochschuldidaktik auf. Deutlich wurde dabei sicherlich, dass eine vertiefende Analyse lohnenswert und erkenntniserweiternd sein würde.

Ausgehend von den beschriebenen Gelingensbedingungen der Kooperation erscheint es sinnvoll, sich mit weiteren theoretisch-konzeptionellen Weiterentwicklungsmöglichkeiten der Verbindung von Hochschuldidaktik und Medienpädagogik an Hochschulen auseinanderzusetzen. Denkbar sind hier Überlegungen zur partizipativen Mediendidaktik von Mayrberger (2019) und zur Kooperationsherstellung (Stolz, im Druck) einerseits, sowie die kritische Analyse von Praxisbeispielen des Jungen Forums Medienund Hochschulforums andererseits (u.a. Barnat u.a. 2013).

Notwendig erscheint weiterhin eine genauere Bestimmung der Begriffe und der vergleichenden Analyse von Aufgaben und Tätigkeitsprofilen. Offen bleibt auch die Frage nach der gegenseitigen Wahrnehmung und Akzeptanz der Berufsfelder. Trotz der gemeinsamen Schnittflächen und Zielvorstellungen mag in der praktischen Arbeit der Eindruck erweckt werden, dass sich «Grabenkämpfe auftun, die noch nicht ausgestanden sind» (Hofhues 2010) und eine Ko-Existenz der Kooperation überwiegt.

Dorothee Meister hätte an dieser Stelle noch einmal deutlich gemacht, welche Bedeutung Allianzen haben und dass es vor allen Dingen um die Erreichung eines gemeinsamen Zieles geht - der innovativen Hochschulentwicklung. Für ihre Weitsicht und Konsequenz möchten wir Dorothee Meister danken. Den Dank sprechen wir Autorinnen stellvertretend für alle (ehemaligen) Mitarbeiter und Mitarbeiterinnen der Stabsstelle Bildungsinnovationen und Hochschuldidaktik aus. 


\section{Literatur}

Barnat, Miriam, Sandra Hofhues, Anne Cornelia Kenneweg, Marianne Merkt, Peter Salden, und Diana Urban. 2013. "Junge Hochschul- und Mediendidaktik: Forschung und Praxis im Dialog». die hochschullehre. Interdisziplinäre Zeitschrift für Studium und Lehre. http://www.hochschullehre.org/?p=396.

Bücker, Diana. i.V. Die Entwicklung der Hochschuldidaktik im Fokus - eine Grounded Theory. Dissertation.

Budde, Jannica, Gudrun Oevel, und Iris Neiske. 2017. «Ausgestaltung einer soziotechnischen Infrastruktur für die Verbreitung und nachhaltige Verankerung von digitaler Lehre». In Informatik 2017, herausgegeben von Maximilian Eibl und Martin Gaedke, 1745-56. Bonn: Gesellschaft für Informatik.

Dany, Sigrid. 2007. Start in die Lehre: Qualifizierung von Lehrenden für den Hochschulalltag. Bildung - Hochschule - Innovation 4. Berlin: LIT.

Eckstein, Brigitte. 1972. Hochschuldidaktik und gesamtgesellschaftliche Konflikte. Frankfurt am Main: Edition suhrkamp.

Gotzen, Susanne, Sylvia Heuchemer, und Timo van Treeck, Hrsg. 2019. Hochschuldidaktik forscht zur Kultur des Ermöglichens. Forschung und Innovation in der Hochschulbildung 3. Zentrum für Lehrentwicklung.

Graf-Schlattmann, Marcel, Dorothee M. Meister, Gudrun Oevel, und Melanie Wilde. 2018. «Hochschulstrategie Als Prozess. Zum Allgemeinen Und Hochschulspezifischen Begriff Der Strategie», Juni. https://doi.org/10.5281/ZENODO.1293797.

Hartung-Griemberg, Anja, und Bernd Schorb. 2017. «Medienpädagogik». In Grundbegriffe Medienpädagogik, herausgegeben von Bernd Schorb, Anja HartungGriemberg und Christine Dallmann. 277-83. München: kopaed.

Hauenschild, Wilfried, Dorothee M. Meister, und Wilhelm Schäfer, Hrsg. 2010. Hochschulentwicklung innovativ gestalten: das Projekt Locomotion an der Universität Paderborn. Medien in der Wissenschaft 53. Münster, New York, Berlin: Waxmann.

Helmke, Andreas, und Friedrich Wilhelm Schrader. 2010. «Hochschuldidaktik». In Handwörterbuch Pädagogische Psychologie, von Detlef H. Rost, 4., überarbeitete und erweiterte Auflage. Weinheim: Beltz.

Hochschulforum Digitalisierung. 2017. «Strategien für Hochschulbildung im digitalen Zeitalter». Hochschulforum Digitalisierung - Hochschulbildung im digitalen Zeitalter. 23. Februar 2017. https://hochschulforumdigitalisierung. de/de/strategien-hochschulbildung-digitales-zeitalter.

Hofhues, Sandra. 2010. «Hochschuldidaktik, E-Learning und Forschungswolke: Eindrücke von der DOSS 2010». Sandra Hofhues - «in the Sky» (blog). 2010. https://www.sandrahofhues.de/2010/03/05/hochschuldidaktik-e-learning-undforschungswolke_eindruecke-von-der-doss-2010/. 
Huber, Ludwig. 1983. "Hochschuldidaktik als Theorie der Bildung und Ausbildung». In Ausbildung und Sozialisation in der Hochschule, herausgegeben von Ludwig Huber und Dieter Lenzen, 114-38. Enzyklopädie Erziehungswissenschaft, Handbuch und Lexikon der Erziehung in 11 Bänden; Bd. 10. Stuttgart: Klett-Cotta.

Huber, Ludwig, und Karin Reiber. 2017. «Hochschule und Hochschuldidaktik im Blick der Erziehungswissenschaft». Erziehungswissenschaft 28 (1): 85-94. https://doi.org/10.3224/ezw.v28i1.10.

Hugger, Kai-Uwe. 2008. «Professionalisierung der Medienpädagogik». In Handbuch Medienpädagogik, herausgegeben von Uwe Sander, Friederike von Gross, und Kai-Uwe Hugger, 559-63. Wiesbaden: VS Verlag für Sozialwissenschaften. https://doi.org/10.1007/978-3-531-91158-8_81.

Hüther, Jürgen, und Bernd Podehl. 2005. «Geschichte der Medienpädagogik». In Grundbegriffe Medienpädagogik, herausgegeben von Jürgen Hüther und Bernd Schorb, 4. Aufl., 116-26. München: kopaed.

Kamin, Anna-Maria, und Dorothee M. Meister. 2015. «Medienpädagogik in Institutionen der Erwachsenen- und Weiterbildung». In Enzyklopädie Erziehungswissenschaft Online, herausgegeben von Dorothee M. Meister, Friederike von Gross, und Uwe Sander, 1-24. Weinheim; Basel: Beltz Juventa. https://doi.org/ 10.3262/EEO18130315.

Mayrberger, Kerstin. 2019. Partizipative Mediendidaktik. Erfurt: Beltz Juventa.

Meister, Dorothee M. 2005. Einflüsse Neuer Medien auf die Weiterbildung: Rahmenbedingungen, System- und Feldadaptation sowie Anforderungen und Potenziale. Bielefeld: Universität Bielefeld. http://nbn-resolving.de/urn:nbn:de:hbz:361-8095.

Meister, Dorothee M. 2018. «Medienpädagogik. Herausforderungen der Digitalisierung». https://www.kulturrat.de/themen/kulturelle-bildung/kulturellebildung-digital/medienpaedagogik/?print=pdf.

Meister, Dorothee M., und Diana Urban. 2014. «Entwicklungen der Hochschuldidaktik - ein vergessenes Feld der Professionsforschung?» In Professionalität: Wissen - Kontext: sozialwissenschaftliche Analysen und pädagogische Reflexionen zur Struktur bildenden und beratenden Handelns; [Festschrift für Prof. Dr. Bernd Dewe], herausgegeben von Martin P. Schwarz, Bernd Dewe, Wilfried Ferchhoff, und Ralf Vollbrecht, 644-62. Bad Heilbrunn: Klinkhardt.

Riegraf, Birgit, Dorothee M. Meister, Peter Reinhold, Niclas Schaper, und Timon T. Temps. 2018. Heterogenität als Chance: Bilanz und Perspektiven des Qualitätspakt Lehre-Projekts an der Universität Paderborn. Paderborn: Universität Paderborn.

Schiefner-Rohs, Mandy, und Michael Kerres. 2012. «Hochschule». In EEO EnzYklopädie Erziehungswissenschaften Online, herausgegeben von Dorothee M. Meister, Friederike von Gross, und Uwe Sander, 1-20. Weinheim; Basel: Beltz Juventa. http://doi.org/10.3262/EEO18120259. 
Scholkmann, Antonia, Sabine Brendel, Tobina Brinker, und Robert Kordts-Freudinger, Hrsg. 2018. Zwischen Qualifizierung Und Weiterbildung: Reflexionen Zur Gekonnten Beruflichkeit in Der Hochschuldidaktik. Blickpunkt Hochschuldidaktik, Thema 134. Bielefeld: wbv Media GmbH \& Co. KG.

Schorb, Bernd. 1995. «Die Geschichte der Medienpädagogik. Eine Bewegung im Zyklus». In Medienalltag und Handeln: Medienpädagogik im Spiegel von Geschichte, Forschung und Praxis. Opladen: VS Verlag für Sozialwissenschaften.

Seufert, Sabine, und Dieter Euler. 2005. «Nachhaltigkeit von eLearningInnovationen: Fallstudien zu Implementierungsstrategien von eLearning als Innovationen an Hochschule». SCIL-Arbeitsbericht. 4. Universität St. Gallen.

Stolz, Katrin. 2020, im Druck. "Hochschuldidaktische Professionalität. Eine Grounded Theory zur Kooperationsherstellung in der Hochschuldidaktik». Dissertation, Magdeburg: Otto-von-Guericke-Universität Magdeburg.

Stolz, Katrin, und Diana Bücker. 2018. «Hochschuldidaktische Professionalität Ausgewählte Facetten Und Implikationen Für Die Praxis». In Zwischen Qualifizierung Und Weiterbildung: Reflexionen Zur Gekonnten Beruflichkeit in Der Hochschuldidaktik, herausgegeben von Antonia Scholkmann, Sabine Brendel, Tobina Brinker, und Robert Kordts-Freudinger, 97-128. Blickpunkt Hochschuldidaktik, Thema 134. Bielefeld: wbv Media GmbH \& Co. KG.

Süss, Daniel, Claudia Lampert, und Christine Wijnen. 2013. Medienpädagogik: ein Studienbuch zur Einführung. 2., überarb. und aktualisierte Aufl. Studienbücher zur Kommunikations- und Medienwissenschaft Lehrbuch. Wiesbaden: Springer VS. https://doi.org/ 10.1007/978-3-531-19045-7.

Szczyrba, Birgit, Sylvia Heuchemer, und Timo van Treeck. 2012. «Qualitätsmanagement in Studium und Lehre mit der Acadamic Balanced Scorecard». Herausgegeben von Winfried Benz, Jürgen Köhler, und Klaus Landfried. Handbuch Qualität in Studium, Lehre und Forschung.

Tulodziecki, Gerhard. 2011. «Zur Entstehung und Entwicklung zentraler Begriffe bei der pädagogischen Auseinandersetzung mit Medien». MedienPädagogik: Zeitschrift für Theorie Und Praxis Der Medienbildung 20 (Medienbildung - Medienkompetenz), 11-39. https://doi.org/10.21240/mpaed/20/2011.09.11.X.

Wildt, Johannes. 2013. «Entwicklung und Potentiale der Hochschuldidaktik». In Professionalisierung der Lehre: Perspektiven formeller und informeller Entwicklung von Lehrkompetenz im Kontext der Hochschulbildung, herausgegeben von Matthias Heiner, Johannes Wildt, und Deutsche Gesellschaft für Hochschuldidaktik, 27-57. Blickpunkt Hochschuldidaktik 123. Bielefeld: Bertelsmann. 